\title{
Molecular characterization of lipoxygenase genes and their expression analysis against biotic and abiotic stresses in Panax ginseng
}

Article in European Journal of Plant Pathology · January 2016

DOI: $10.1007 /$ s10658-015-0847-9

\section{CITATION}

1

9 authors, including:

Srirenukadevi Balusamy

Seoul National University

16 PUBLICATIONS 98 CITATIONS

SEE PROFILE

\section{Johan Sukweenadhi}

Kyung Hee University

19 PUBLICATIONS 46 CITATIONS

SEE PROFILE

\section{Altanzul Khorolragchaa}

Mongolian Academy of Sciences

18 PUBLICATIONS 116 CITATIONS

SEE PROFILE

\section{Deok-Chun Yang}

Kyung Hee University

538 PUBLICATIONS 3,334 CITATIONS

SEE PROFILE 
Molecular characterization of lipoxygenase genes and their expression analysis against biotic and abiotic stresses in Panax ginseng

Kwi-Sik Bae, Shadi Rahimi, Yu-Jin Kim, Balusamy Sri Renuka Devi, Altanzul Khorolragchaa, Johan Sukweenadhi, Jeniffer Silva, et al.

European Journal of Plant Pathology Published in cooperation with the European Foundation for Plant Pathology

ISSN 0929-1873

Eur J Plant Pathol DOI 10.1007/s10658-015-0847-9
Volume 144 No. 2 February 2016 ONLINE FIRST

Suropean Journal of Plant Pathology

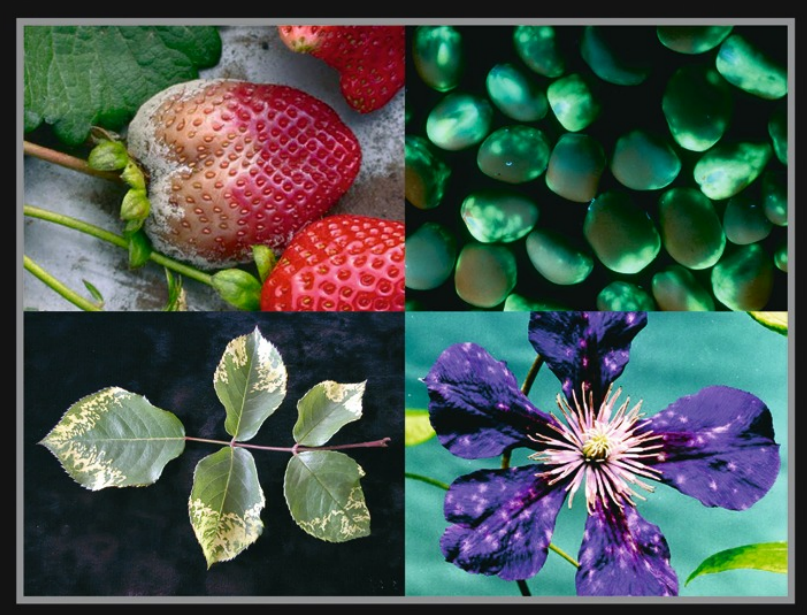

Springer In cooperation with European Foundation for Plant Pathology 
Your article is protected by copyright and all rights are held exclusively by Koninklijke Nederlandse Planteziektenkundige Vereniging. This e-offprint is for personal use only and shall not be self-archived in electronic repositories. If you wish to self-archive your article, please use the accepted manuscript version for posting on your own website. You may further deposit the accepted manuscript version in any repository, provided it is only made publicly available 12 months after official publication or later and provided acknowledgement is given to the original source of publication and a link is inserted to the published article on Springer's website. The link must be accompanied by the following text: "The final publication is available at link.springer.com". 


\title{
Molecular characterization of lipoxygenase genes and their expression analysis against biotic and abiotic stresses in Panax ginseng
}

\author{
Kwi-Sik Bae • Shadi Rahimi • Yu-Jin Kim • Balusamy Sri Renuka Devi • \\ Altanzul Khorolragchaa • Johan Sukweenadhi • Jeniffer Silva • \\ Davaajargal Myagmarjav • Deok-Chun Yang
}

Accepted: 18 December 2015

(C) Koninklijke Nederlandse Planteziektenkundige Vereniging 2016

\begin{abstract}
Lipoxygenase (LOX) belongs to a family of non-heme-iron-containing fatty acid dioxygenases that are widely distributed in plants and animals. LOX involved in the synthesis of jasmonic acid and six-carbon (C6) volatiles which is necessary for plant growth and responses to a wide range of biotic and abiotic stresses. We have isolated and characterized LOX cDNA clones from Panax ginseng Meyer. From their deduced amino acid sequences, two diverse classes of 9-LOX (LOX1, LOX2, and LOX3) and 13-LOX (LOX4, LOX5) are defined in P. ginseng. A GenBank Blast X search revealed that the deduced amino acid of PgLOXs share a high degree of homology with LOX from other plants and mammals especially in three distinct motifs; motifl harboring iron binding regions, motif 2 and motif3.
\end{abstract}

Kwi-Sik Bae and Shadi Rahimi contributed equally to this work.

Electronic supplementary material The online version of this article (doi:10.1007/s10658-015-0847-9) contains supplementary material, which is available to authorized users.

D.-C. Yang

Department of Oriental Medicinal Biotechnology, College of Life Sciences, Kyung Hee University, Yongin 446-701,

Republic of Korea

K.-S. Bae • S. Rahimi $(\bowtie) \cdot$ Y.-J. Kim • B. S. R. Devi •

A. Khorolragchaa · J. Sukweenadhi · J. Silva •

D. Myagmarjav · D.-C. Yang $(\bowtie)$

Graduate School of Biotechnology and Ginseng Bank,

College of Life Sciences, Kyung Hee University,

Yongin 446-701, Republic of Korea

e-mail: shadirahimi@khu.ac.kr

e-mail: dcyang@khu.ac.kr
Chloroplast localization was predicted for PgLOX5. $P g L O X S$ displayed organ-specific expression, highly expressed in aerial parts of the plant such as 3-year old flower, stem and leaf tissues. $P g L O X s$ mRNAs were elevated strongly by bacterial infection. Expression of $P g L O X s$ was differentially induced in ginseng not only by mechanical damage and methyl jasmonate but also after exposure to withholding water. Ginseng 13-LOXs positively respond to wounding that may involve in production of C6 volatiles and jasmonic acid at the wounded sites. However, the higher expression of PgLOX3 by water deficit and $82 \%$ of the nucleotide sequence identity with the EST from severe drought-stressed leaves of Populus (CU229089.1) at +6371 bp downstream of $P g L O X 3$ genomic DNA structure can suggest drought tolerance role for $P g L O X 3$. Ginseng LOX genes have different expression pattern which may suggest different specific function against various environmental stresses.

Keywords Abiotic stress · Biotic stress · Gene expression · Jasmonic acid · Lipoxygenase · Panax ginseng
Abbreviations
AOC allene oxide cyclase
AOS allene oxide synthase
EST expressed sequence tag
HPL hydroperoxide lyase
13-HPOD (13S)-hydroperoxyoctadecadienoic acid
9-HPOD (9S)-hydroperoxyoctadecadienoic acid
IBA indole-3-butyric acid 


\begin{tabular}{|c|c|}
\hline $\mathrm{JA}$ & jasmonic acid \\
\hline LOX & lipoxygenase \\
\hline MJ & methyl jasmonate \\
\hline OPDA & (9S,13S)-12-oxo phytodienoic acid \\
\hline Pst & $\begin{array}{l}\text { Pseudomonas syringae pv tomato strain } \\
\text { DC } 3000\end{array}$ \\
\hline UFAs & polyunsaturated fatty acids \\
\hline T-PCR & $\begin{array}{l}\text { reverse transcriptase-polymerase chain } \\
\text { reaction. }\end{array}$ \\
\hline
\end{tabular}

\section{Introduction}

Panax ginseng Meyer, a perennial herbaceous plant from Araliaceae family, is one of the most commonly utilized medicinal plants. Due to long cultivation period of ginseng, various ecological stresses might affect the plant and this condition makes this highly valued medicinal plant more susceptible to environmental stresses. However, plants activate distinct defense responses which are mediated by a complex hormonal network to adapt with adverse conditions during their growth and development. Jasmonates belong to the oxygenated compounds known as oxylipins are essential signaling molecules in response to environmental changes as well as growth and development. Oxylipin biosynthesis is initiated by the function of LOX enzyme (EC1.13.11.12) which is ubiquitously occurring in plant and mammals and catalyzed hydroperoxidation of polyunsaturated fatty acids. Interestingly, LOX-derived oxylipins are involved in wound healing and defense processes in plants, while in mammals they are involved in inflammation, asthma and heart disease (Andreou and Feussner 2009).

Two different isomer products of 13-HPOD and 9HPOD are synthesized by introduction of molecular oxygen either at carbon atom 9 or at carbon atom 13 of hydrocarbon backbone by the function of plant LOX which can justify their role as 13-LOX and 9-LOX, respectively (Wasternack 2007). LOX function can change from 13-to 9-LOX by a change in histidine residue of substrate binding site (Hornung et al. 1999). 9-LOXs are a subfamily of proteins which share high amino-acid sequence identity $(\sim 60 \%)$ to one another, but 13 -LOXs are sharing only $\sim 35 \%$ sequence identity among themselves (Andreou and Feussner 2009; Park et al. 2013). The discrete pathways are proposed for 13LOX and 9-LOX which can lead to numerous types of oxylipins. 13-HPOD can be further metabolized into jasmonates in plants. 13-LOX and 9-LOX can be differentiated by their gene expression patterns and subcellular localizations (Shen et al. 2014). LOX form gene families ranging from six in Arabidopsis to at least 18 genes in potato which differently localized in microsomal membranes (Feussner and Kindl 1994), plasma membranes (Nellen et al. 1995), and lipid bodies (Feussner and Kindl 1992) of cucumber cotyledons, cytosol and vacuole of soybean leaves (Grayburn et al. 1991; Stephenson et al. 1998), envelope of leaf chloroplasts in spinach, barley, tomato, potato or Arabidopsis (Blée and Joyard 1996; Feussner et al. 1995; Heitz et al. 1997; Royo et al. 1996). Different LOXs may have different function in plants. There are different four major fates for the LOX-derived hydro (pero) xy PUFAs in plants (Feussner and Wasternack 2002). First is hydroperoxide isomerase pathway which converts fatty acid hydroperoxides to epoxy- or dihydrodiol polyenoic fatty acids, second is AOS pathway which forms unstable allene oxides further metabolized to OPDA by an AOC. Third is the HPL pathway oxidatively cleaves the hydrocarbon backbone of fatty acid hydroperoxides and forms short chain aldehydes (C6- or C9-). Fourth one is the DES pathway forms divinyl ethers such as colneleic acid or colnelenic acid. Ketodiene-forming pathway, epoxy alcohol synthase pathway and reductase pathway are other reactions for hydro (pero) xy PUFAs metabolisms which is less studied.

Since JA will be rapidly accumulated in response to wounding or herbivore attack to confer direct defenses at the site of attack and systemic defense signaling (Howe and Jander 2008), the characterization of LOX as the critical protein catalyzed the initial step of JA pathway will help us to understand the plant defense mechanism in response to ecological stresses. Despite of the importance of LOX, there is a dearth of genetic evidence for the function of this defensive gene in the ginseng plant. On the one hand, weeds compete with ginseng to absorb water and nutrients and on the other hand, due to the long cultivation period, ginseng is frequently exposed to bacterial infection and herbivores. In these stressful conditions, ginseng root growth will be affected and it can decrease the worth of this economically important plant.

In this report, we attempt to identify the five LOX genes in $P$. ginseng and understand the biological significance of these genes in the initial step of plant 
interaction with pathogens, wounding and in drought condition. In addition, the expression of these LOX genes at different organs was examined. The present study examined the phylogenetic relationship of ginseng LOXs and that of other organisms, to contribute to the understanding of the possible role of ginseng LOXs.

\section{Materials and methods}

Identification of the LOX genes from the $P$. ginseng EST database

To identify genes from the EST library, we used the EST library constructed from $P$. ginseng (Sathiyamoorthy et al. 2009). Homologous sequences of LOX ESTs were searched against the GenBank databases using a BLASTX algorithm. We identified and selected the LOX genes based on open reading frames encoding the specific protein via the BlastX program (NCBI BlastX program). Clustal $\mathrm{X}$ with default gap penalties was used to perform multiple alignments of LOXs isolated from $P$. ginseng and previously registered in other species. A phylogenetic tree was constructed by the neighbor-joining method, and the reliability of each node was established by bootstrap methods using MEGA6 software. The protein properties were estimated using ProtParam (Gasteiger et al. 2005) and the hydropathy value was calculated by the method described by Kyte and Doolittle (1982). Identification of conserved motifs within LOXs was accomplished with MEME (Bailey et al. 2009). Chloroplast localization was inspected by ChloroP (Emanuelsson et al. 1999) and proposed signal peptide is predicted from PSORT (K. Nakai, Tokyo University, http://psort.ims.u-tokyo. ac.jp/). Other database also used to analyze the $P g L O X$ genes, such as MotifScan (http://myhits.isbsib.ch/cgibin/motif_scan), HMMTOP (http://www.enzim.hu/ hmmtop), SOPMA (http://npsa-pbil.ibcp.fr/cgi-bin/ npsa_automat.pl?page=/NPSA/npsa_server.html).

Plant materials and growth conditions

P. ginseng adventitious roots were collected from Ginseng Bank, Kyung Hee University and grown for 1 month in liquid Murashige \& Skoog medium (Murashige and Skoog 1962) supplemented with $2 \mathrm{mg} \mathrm{L}^{-1} \mathrm{IBA}$ at $25^{\circ} \mathrm{C}$. The roots were maintained by regular subculture every 4 weeks. Different organs of 3- year old healthy ginseng plants (leaf, stem, flower bud, and root) were collected from Ginseng Bank, Korea.

Stress treatment

To investigate the response of the $P g L O X$ genes to MJ elicitor, $100 \mu \mathrm{M} \mathrm{MJ}$, was applied to 30-day-old subcultured adventitious roots, and harvested at 6,12 , $24,48,72 \mathrm{~h}$ after treatment. The $P$. ginseng seeds used in the present study were obtained from the Korean Ginseng Resource Bank, South Korea. The 4- weekold ginseng seedlings germinated in in vitro condition on solidified MS media (Duchefa biochem, Netherland) with $3 \%$ sucrose, supplemented with gibberellic acid $\left(10 \mathrm{mg} \mathrm{L}^{-1}\right.$ ) at $25^{\circ} \mathrm{C}$ under a $16 \mathrm{~h}$ photoperiod and used for the treatment studies. To induce drought stress, plants were exposed to dehydration by withholding water. Ginseng plants were inoculated with the virulent Pseudomonas syringae pv tomato (Pst) strain DC3000. The bacteria were grown at $28^{\circ} \mathrm{C}$ in King's B medium containing rifampicin $\left(50 \mathrm{mg} \mathrm{L}^{-1}\right)$. To study bacterial infection, bacterial suspensions $\left(5 \times 10^{6} \mathrm{cfu} \mathrm{mL}^{-1}\right)$ were infiltrated into the ginseng leaves using a syringe. At appropriate time points, three independent leaves infiltrated with Pst were harvested. For wounding experiments, leaves were pricked with a needle (Hwang and Hwang 2010). For stress treatments, the plantlets were subjected to treatment for $6,12,24,48$ and $72 \mathrm{~h}$. Control plants were grown at $25^{\circ} \mathrm{C}$ under a $16 \mathrm{~h}$ photoperiod.

RNA extraction and quantitative RT-PCR analysis

Total RNA was extracted from adventitious roots of $P$. ginseng using RNeasy mini kit (Qiagen, Valencia, CA, USA). For RT-PCR, 200 ng of total RNA was used as a template for reverse transcription using oligo (dT) 15 primer $(0.2 \mathrm{mM})$ and AMV Reverse Transcriptase (10 $\mathrm{U}_{\mu \mathrm{L}^{-1}}$ ) (INTRON Biotechnology, Inc., South Korea) according to the manufacturer's instructions. Real-time quantitative PCR was performed using 100 ng of cDNA in a $10-\mu \mathrm{L}$ reaction volume using $\mathrm{SYBR}^{\circledR}$ Green Sensimix Plus Master Mix (Quantace, Watford, England). Gene-specific primers listed in Table 1, were used to perform quantitative RT-PCR. The thermal cycler conditions recommended by the manufacturer were used as follows: $10 \mathrm{~min}$ at $95^{\circ} \mathrm{C}$, followed 40 cycles of $95{ }^{\circ} \mathrm{C}$ for $10 \mathrm{~s}, 58{ }^{\circ} \mathrm{C}$ for $10 \mathrm{~s}$, and $72{ }^{\circ} \mathrm{C} 20 \mathrm{~s}$. The fluorescent product was detected at the last step of each cycle. Relative quantity of the $\operatorname{PgLOX}$ genes 
Table 1 List of gene-specific primer sequences used for qRTPCR analysis

\begin{tabular}{ll}
\hline Gene & Primers $\left(5^{\prime}-3\right.$ ') \\
\hline$P g L O X 1$ & F: ATCAAGAGAGGGATGGCAGTTGA \\
& R: ATGGCCCTTTTCTCGGACTTCT \\
$P g L O X 2$ & F: TACTGATCGAGGACTACCCATATGCA \\
& R: CATTTTAGGCCATGCTTCAT \\
$P g L O X 3$ & F: ATGTTGCCATTTCTGCTCAAGTTC \\
& R: CAGATATCCAACCACGACAAGCA \\
$P g L O X 4$ & F: CAAGTCATTCTTACCATCTCAAAC \\
$P g L O X 5$ & R: CCTTTAGTGTGAACGATGTCTCCT \\
& F: TATGTGCCTAATCGTCCCACTCTCAT \\
$P g \beta$-actin & R: CCTGAACCAAGTACTCTTCATCTGG \\
& F: GTGATCTTACAGATAGCTTGATGA \\
& R: AGAGAAGCTAAGATTGATCCTCC \\
\hline
\end{tabular}

transcription level was performed using Rotor-Gene 6000 real-time rotary analyzer (Corbett Life Science, Sydney, Australia), and calculated using the comparative cycle threshold (CT) method according to the manufacturers' instructions for normalizing data. A constitutively expressed $\beta$-actin gene was used as internal reference. Three independent experiments were performed. The primer efficiencies were determined according to the method by Livak and Schmittgen (2001) to validate the $\Delta \Delta \mathrm{Ct}$ method. The observed slopes were close to zero, indicating that the efficiencies of the gene and the internal control $\beta$-actin were equal.

\section{Results}

Isolation and characterization of $P g L O X$ genes

As the part of a genomic project to identify genes of the medicinal plant $P$. ginseng, a cDNA library consisting about 20,000 cDNAs were previously constructed. Five LOXs, designated PgLOX1, PgLOX2, PgLOX3, $P g L O X 4$, and $P g L O X 5$ were isolated. Moreover, full genomic DNA sequence of $P g L O X s$ was analyzed using the genomic DNA sequence retrieved from ginseng genome database (http://im-crop.snu.ac.kr/new/index. php). The corresponding characteristics of each PgLOXs are indicated in Table 2. As it was shown in Fig. 1a GenBank Blast search (http://www.ncbi.nlm. nih.gov/BLAST/) resulting in highly conserved Nterminus domain of PLAT/LH2 which is found in a variety of membrane or lipid associated proteins to possibly mediate interaction with lipids or membrane bound proteins and the C-terminus domain of LOX superfamily possessed by all the previously cloned plant and indicated that PgLOX1,PgLOX2,PgLOX3, $P g L O X 4$, and $P g L O X 5$ belonged to the LOX family. Red underlined sequence showed the proposed signal peptide in Fig. 1 predicted by IPSORT program, showing that PgLOX5 had a transit peptide at its N-terminal which may suggest chloroplast localization. Multiple sequence alignment revealed that the N-terminal sequences differ among the LOXs in different plants, including five PgLOXs (Fig. 1). Plant LOX enzymes contain iron atom in their active site which coordinated by 5 five amino acids including three histidines, one asparagine and the carboxy group of the carboxyterminal isoleucine. Two iron-binding regions were shown in green boxes in motifl. In addition, three conserved motifs were found by MEME analysis in all plant LOX isozymes and mammals (Fig. 2b) Three conserved motif sequences predicted by MEME are shown in Online Resource 1.

It was revealed that the deduced amino acid of $P g L O X 1, P g L O X 2$ and PgLOX3 share a high degree of sequence homology with 9-LOX proteins encoded by Actinidia deliciosa (ABF60002.1) (identity $74 \%$ \& similarity $99 \%$ ), Camellia sinensis (ABW75772.2) (identity $79 \%$ \& similarity $100 \%$ ), Capsicum annuum (ABF19103.2) (identity $78 \%$ \& similarity $98 \%$ ), respectively. However, PgLOX4 and PgLOX5 share a high degree of sequence homology with chloroplastic 13-LOX proteins encoded by Camellia sinensis (ADO51752.1) (identity $66 \%$ \&similarity $100 \%$ ) and Theobroma cacao (XP_007045017.1) (identity 78 \% \& similarity $90 \%$ ), respectively. Clustal $X$ and the MEGA6 Program were used for the construction of phylogenetic tree based on LOX amino acid sequences (Fig. 2). Moreover, the phylogenetic relationship is shown among LOXs from $P$. ginseng and Arabidopsis thaliana (Online Resource 2). Transmembrane helices of integral membrane proteins and localization of the $\mathrm{N}$ terminus were predicted by HMMTOP and mentioned in Table 2. The hydrophobicity profile of the estimated 9- and 13-LOX proteins is shown in Fig. 3a, b. Nterminal peptides differ from each other but more hydrophilic amino acid composition similar to other LOXs were found in PgLOXs.

Secondary structure analysis and molecular modeling for PgLOXs were conducted by SOPMA (Geourjon and Deleage 1995) (Table 3). The secondary structure 
Table 2 Characteristics of ginseng LOXs

\begin{tabular}{|c|c|c|c|c|c|c|}
\hline Protein & Length $^{\mathrm{a}}$ & Molecular mass $^{\mathrm{a}}$ & $\mathrm{pI}^{\mathrm{a}}$ & $\begin{array}{l}\text { Predicted } \\
\text { localization }^{\mathrm{b}}\end{array}$ & $\begin{array}{l}\text { Transmembrane } \\
\text { helices }^{\mathrm{c}}\end{array}$ & $\begin{array}{l}\text { localization } \\
\text { of } \mathrm{N} \text { terminus }\end{array}$ \\
\hline PgLOX1 & 815 & 94 & 5.4 & - & - & Out \\
\hline PgLOX2 & 860 & 97.7 & 5.8 & - & - & Out \\
\hline PgLOX3 & 868 & 99.3 & 6.5 & - & - & Out \\
\hline PgLOX4 & 762 & 86.6 & 5.2 & - & - & Out \\
\hline PgLOX5 & 908 & 103.4 & 8.2 & Chloroplast & - & Out \\
\hline
\end{tabular}

${ }^{a}$ Length (number of amino acid residues), molecular mass $(\mathrm{kDa})$, and isoelectric point ( $\mathrm{pl}$ ) of PgLOX proteins deduced from the open reading frames (Table 2). Values for predicted mature proteins are indicated in brackets

b Transit peptides of PgLOX proteins were predicted by the IPSORT

c Transmembrane helices of integral membrane proteins and localization of the N-terminus were predicted by HMMTOP

analysis revealed that PgLOX1 consists of $312 \alpha$-helices, $74 \beta$-turns joined by 128 extended strands, and 301 randomcoils; PgLOX4 consists of $268 \alpha$-helices, $73 \beta$ turns joined by 143 extended strands, and 278 randomcoils. PgLOX5 consists of $335 \alpha$-helices, 77 $\beta$-turns joined by 144 extended strands, and 352 randomcoils which is highly similar to the secondary structure of At13-LOX6 from A. thaliana, includes 346 $\alpha$-helices, $62 \beta$-turns joined by 140 extended strands, and 369 random coils; and At9-LOX from A. thaliana, made up of $335 \alpha$-helices, $70 \beta$-turns joined by 149 extended strands, and 332 randomcoils. Similar secondary structure were found in PgLOX2 and PgLOX3 which consists of $297 \alpha$-helices, $90 \beta$-turns joined by 146 extended strands, and 327 randomcoils and $294 \alpha$ helices, $95 \beta$-turns joined by 141 extended strands, and 338 randomcoils, respectively.

Distribution of LOX genes transcript in different organs

To determine the expression of $P g L O X$ genes at different various organs of ginseng, we performed quantitative RT-PCR expression analysis of $\mathrm{PgLOX}$ genes in 3year-old leaf, stem, flower, and root samples (Fig. 4). The results indicate that these LOX enzymes are tissueregulated showing $P g L O X 1, P g L O X 4$, and $P g L O X 5$ transcripts were highly detected in flower bud whereas relatively higher levels of $P g L O X 2$ and $P g L O X 3$ transcripts were observed in leaf and stem, respectively. Three-year-old root tissue contain lowest level of $P g L O X 1, P g L O X 4$ and $P g L O X 5$ transcripts when $P g L O X 3$ transcriptome level appears to be same as that in leaf root tissue.
Expression of PgLOXs in response to MJ elicitor, wounding and bacterial infection

To determine the expression of the $P g L O X$ genes in response to $\mathrm{MJ}$, we performed quantitative RT-PCR analysis of $L O X S$ in ginseng adventitious roots treated with $100 \mu \mathrm{M}$ MJ (Fig. 5). MJ elicitor upregulated PgLOX5 $6 \mathrm{~h}$ after treatment however, PgLOX4 and $P g L O X 3$ were highly responsive $12 \mathrm{~h}$ and $72 \mathrm{~h}$ after MJ treatment. PgLOX1, PgLOX2 and PgLOX3 expression were not responsive to mechanical damage. $P g L O X 4$ was highly upregulated $72 \mathrm{~h}$ after wounding treatment however, $P g L O X 5$ was showed higher transcript level at $48 \mathrm{~h}$ and then it was declined after $72 \mathrm{~h}$.

To recognize pathogen can induce the transcriptome profile of $L O X s$, we infected 30-day old ginseng seedlings with Pseudomonas syringe pv tomato strain DC3000 suspension and checked the transcription pattern of these genes using quantitative RT-PCR analysis. The expression level of all $\mathrm{PgLOXS}$ showed similar pattern with maximum transcription at $48 \mathrm{~h}$ after bacterial infection followed by decreased expression $72 \mathrm{~h}$ after bacterial treatment. The mRNA level of PgLOX2 was induced by pathogen attack however it was lower than other PgLOXs.

\section{Expression of $P g L O X S$ in response to drought stress}

To investigate the possible role of LOXs in response to drought stress, plants were exposed to dehydration by withholding water. The expression patterns of $\mathrm{PgLOXs}$ at different time points after treatments were analyzed using real-time PCR. Among all PgLOXs, only PgLOX3 
was responsive to water shortage. The expression profile of $P g L O X 3$ highly increased at $24 \mathrm{~h}$ of post treatment with drought and after that declined at $48 \mathrm{~h}$ and $72 \mathrm{~h}$ after treatment compared to the control (Fig. 5). The full genomic DNA sequence of $P g L O X 3$ has a length of 2,604 bp encoding 906 amino acids. This gene contains nine exons interrupted by the eight introns (Online Resource 3A). By carefully checking the genomic DNA structure of $P g L O X 3$, a nucleotide sequence with 438 bp size was found at $+6,371-+6,808$ bp downstream of $P g L O X 3$ genomic DNA structure that shares the highest nucleotide sequence identity ( $82 \%$ ) with the EST from severe drought-stressed leaves of Populus (CU229089.1). A GenBank Blast X search revealed that the deduced amino acid of this sequence share a high degree of homology with Photosystem I reaction center subunit D-2 (PsaD2) from other plants (Online Resource 3B) which its Arabidopsis homolog (NP_171812.1) is highly expressed $24 \mathrm{~h}$ after drought treatment.

\section{Discussion}

In our experimental study, for the first time we have isolated and characterized the LOX genes in $P$. ginseng. These sequences were named $P g L O X$ due to their high homology with the LOX sequences of other plants. It was revealed that the deduced amino acid of $P g L O X 1$, $P g L O X 2$ and $P g L O X 3$ shares a high degree of sequence homology with 9-LOX proteins however, that of PgLOX4 and PgLOX5 shares a high degree of sequence homology with chloroplastic 13-LOX proteins. Phylogenic tree analysis of LOXs from different species suggested that the ginseng LOXs are classified into two groups of 9-LOX and 13-LOX with respect to their positional specificity of fatty acid oxygenation. Plant LOX genes encoded around 94-100 kDa protein sequences which contain two different domains. PLAT domain around $25-30 \mathrm{kDa}$ at the $\mathrm{N}$-terminus consists of b-barrel domain (Corbin et al. 2007) and possibly mediates interaction with lipids or membrane bound proteins. There are around $55-65 \mathrm{kDa}$ domain at the C-terminus which conatins a-helices and includes catalytic site of enzyme (Andreou, and Feussner 2009). LOXs belong to a family of non-heme-iron-containing fatty acid dioxygenases that are widely distributed in plants and animals. Two iron binding regions were identified in PgLOX enzymes. PgLOX5 contain transit peptide at its N-terminal which directed the enzyme to
Fig. 1 Multiple amino acid sequence alignment of $P g L O X S$ with those of proteins encoded by Actinidia deliciosa (ABF60002.1), Camellia sinensis (ABW75772.2), Capsicum annuum (ABF19103.2), Camellia sinensis (ADO51752.1) and Theobroma cacao (XP_007045017.1). A hyphen was inserted within the amino acid sequence to denote a gap. Shadow box indicates well conserved residues, * represents a conserved amino acid, and: represents a very similar amino acid. Red underlined sequences are the proposed signal peptide predicted by IPSORT program. The arrow indicates the conserved domains. Three conserved motifs obtained by MEME analysis. Two iron-binding regions are shown in green boxes

the chloroplast. However, there was no homology among the N-terminals of the LOX. Surprisingly, Nterminal chloroplast transit peptide was not identified in 13-LOX ginseng LOX4. However, there are some examples for occurrence of enzymes within or at the plastid without transit peptide. Despite the lack of a putative chloroplast signal peptide in AOS sequences of barely, immunocytochemical analysis showed their chloroplast localization (Maucher et al. 2000). Those chloroplast proteins without transit peptide are located in outer envelope membrane of chloroplast which imported through the transit peptide-independent path (Keegstra and Cline 1999).

The temporal expression analysis from different organs demonstrated that $P g L O X$ mRNAs were ubiquitously expressed in all organs that we examined though an expression level of $L O X S$ is low in root tissue. $L O X S$ were strongly expressed in aerial parts of the plant, especially in 3-year old flower, stem and leaf tissues. Since, JA is involved in regulation of pollen maturation, anther dehiscence, and flower opening (Scott et Al. 2004); we can suggest that higher expression of LOXs in ginseng flower will provide evidence for their possible role in producing JA during flower development.

Ginseng plants have evolved effective mechanisms to deal with the ever-changing environment during their growth and development. Understanding these mechanisms is helpful to improve stress tolerance in ginseng plant. In order to determine the molecular mechanisms of ginseng plant adapted to biotic and abiotic challenges over long years of life cycle, transcript profiling of $P g L O X s$ in response to wounding, MJ supplementation, pathogen attack and drought was studied by quantitative real time-PCR analysis. $L O X$ expression in plants is regulated in response to stresses. In our current study, ginseng $L O X S$ transcript level exhibited different results. $L O X 1, L O X 2$ and LOX3 classified as the 9-LOXs, were 

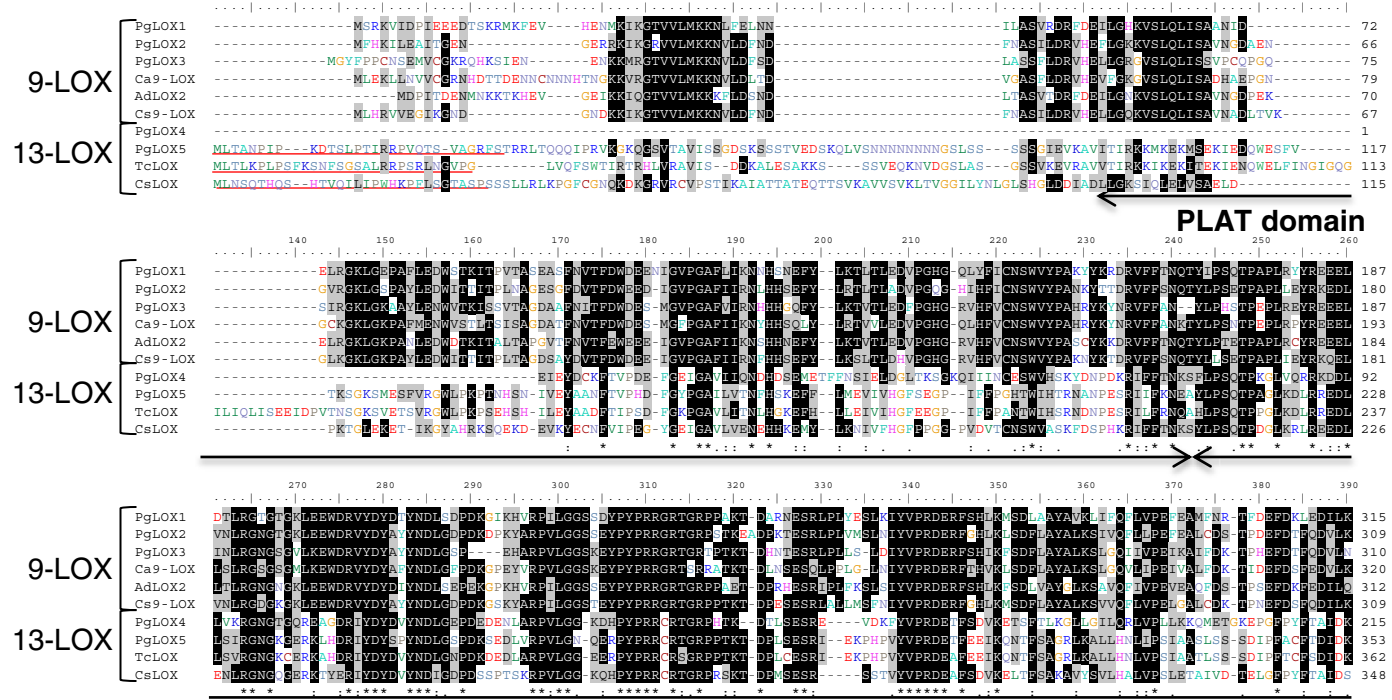

\section{Lipoxygenase iron-binding catalytic domain}
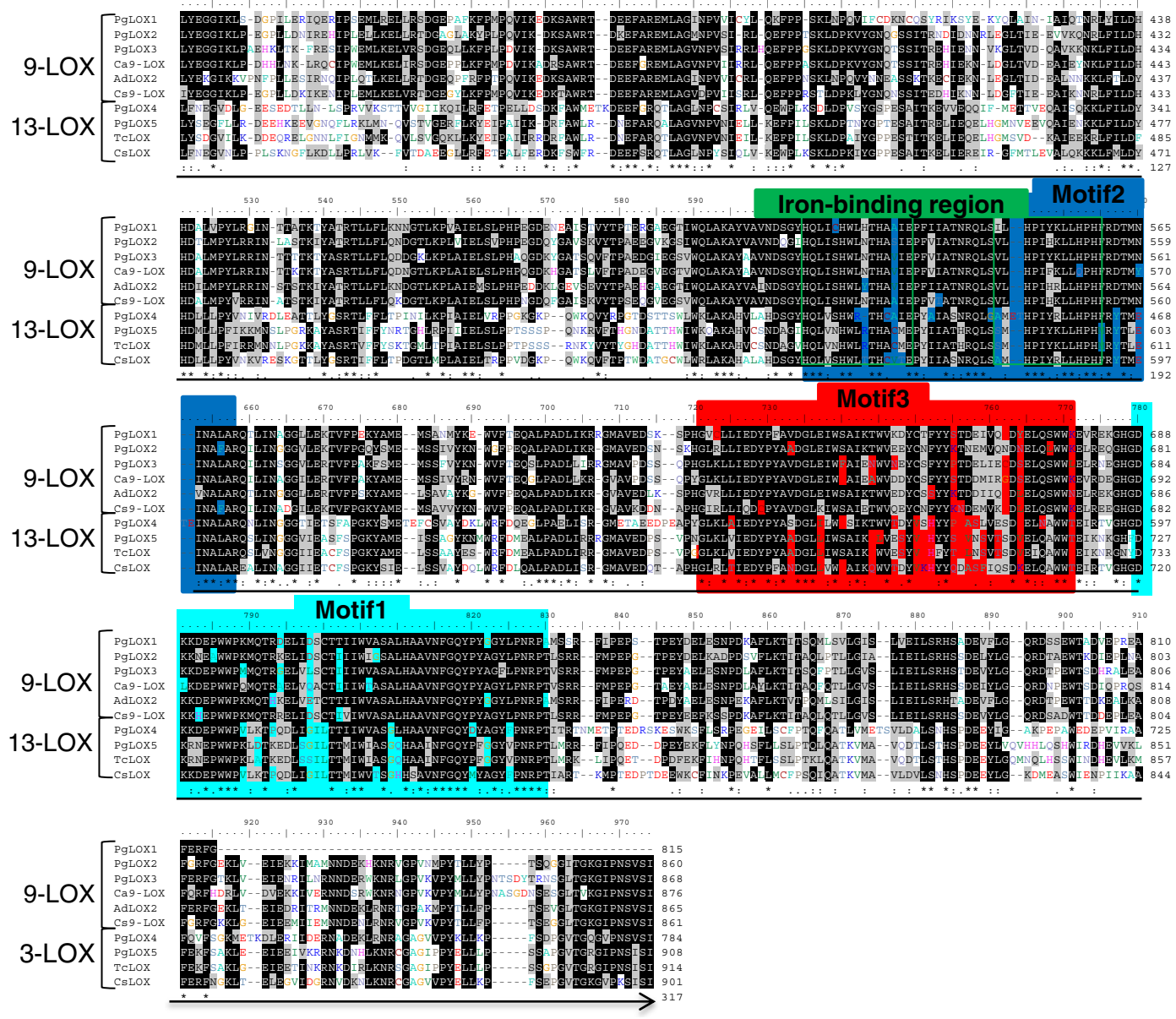

not responsive to wounding which is in consistent with the previous report by Yang et al. (2012) showed the downregulation of cucumber LOX in response to wounding. Meanwhile, LOX4 and LOX5 which belong 
a

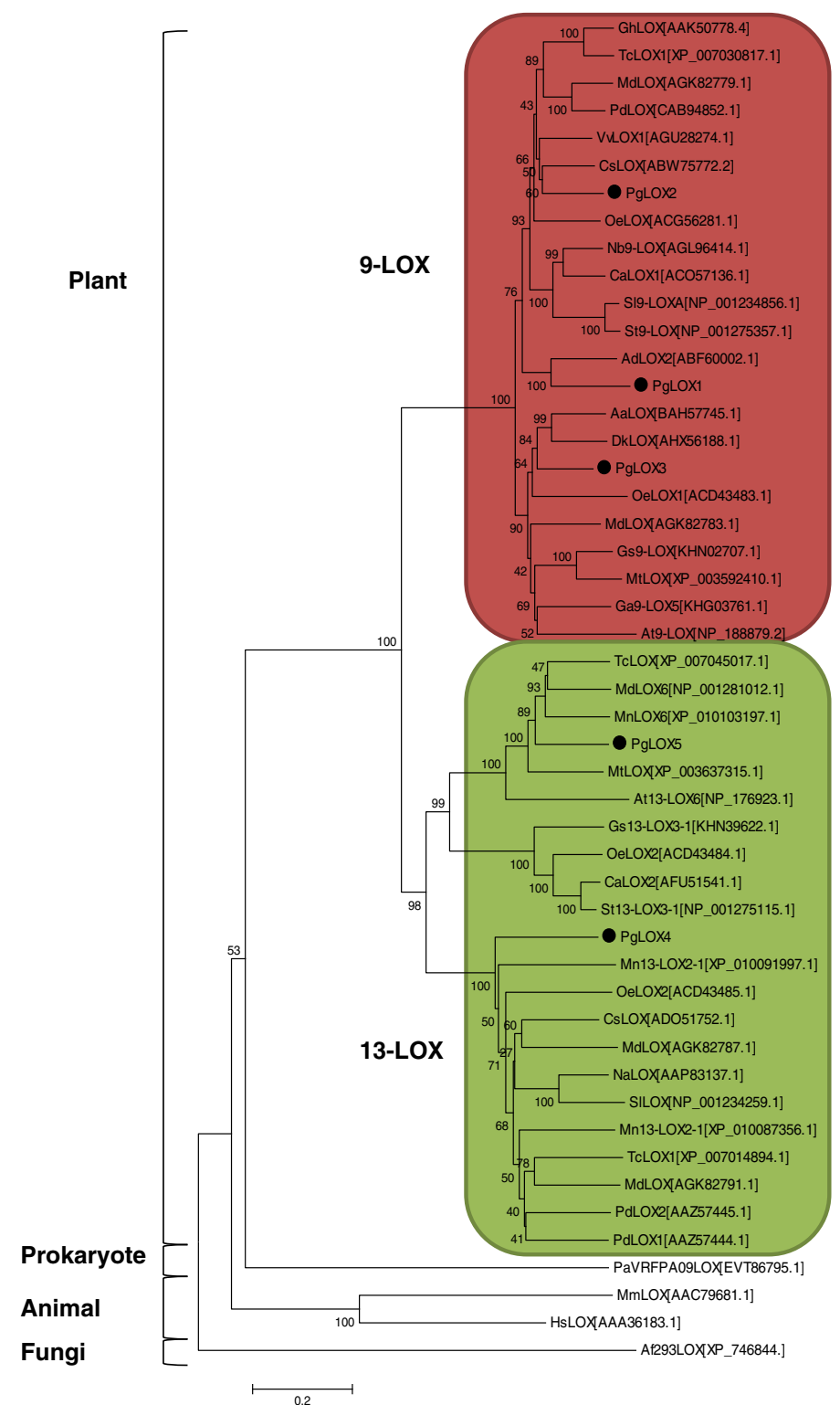

b

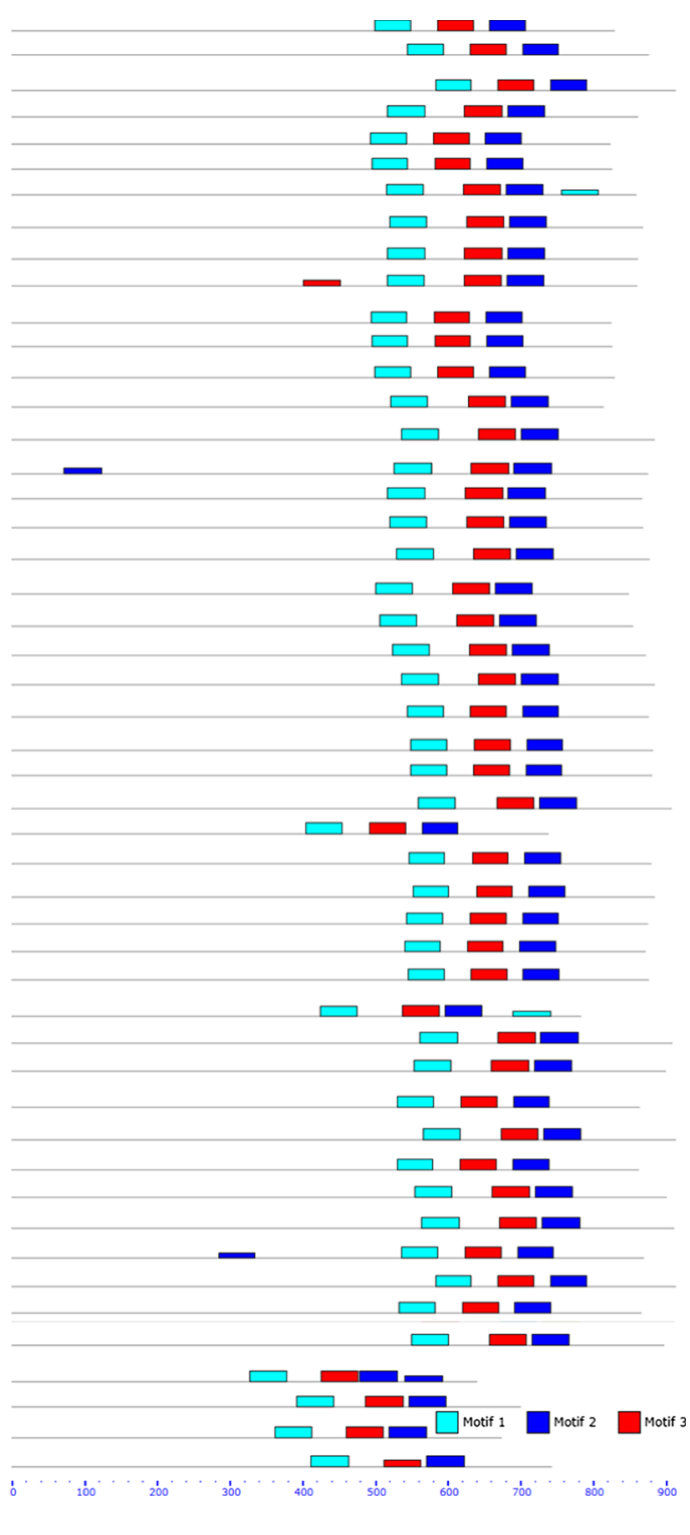

Fig. 2 Sequence homology analysis of PgLOXs with other LOX genes. a Phylogenetic relationships in LOX from various organisms including plant species. Database accession numbers are indicated in brackets. Distances between each clone and group are calculated with Clustal X program. 9-LOX and 13-LOX are

to 13-LOXs, were highly induced in mechanically damaged ginseng seedlings and it can be correlated to their specific function in production of related oxylipins which can help to relieve the tension. Three potential fates for LOX-derived hydroperoxides were identified by Shen et al. (2014). One of them is shown in red and green boxes, respectively. b Organization of putative motifs in LOXs identified by MEME. Numbered color boxes represent different putative motifs Motifs 1, 2, and 3 are indicated by the mint (First), blue (middle), and red (last) boxes, respectively. Motif sizes are indicated at the bottom of the figure
JA pathway and its first step catalyzed by allene oxide synthase. The other one is biosynthesized C6 volatiles which initiated by oxidative cleavage of hydroperoxides through the action of HPL. The third pathway is the C5 compounds synthesis and in that LOX oxygenate hydroperoxides. 

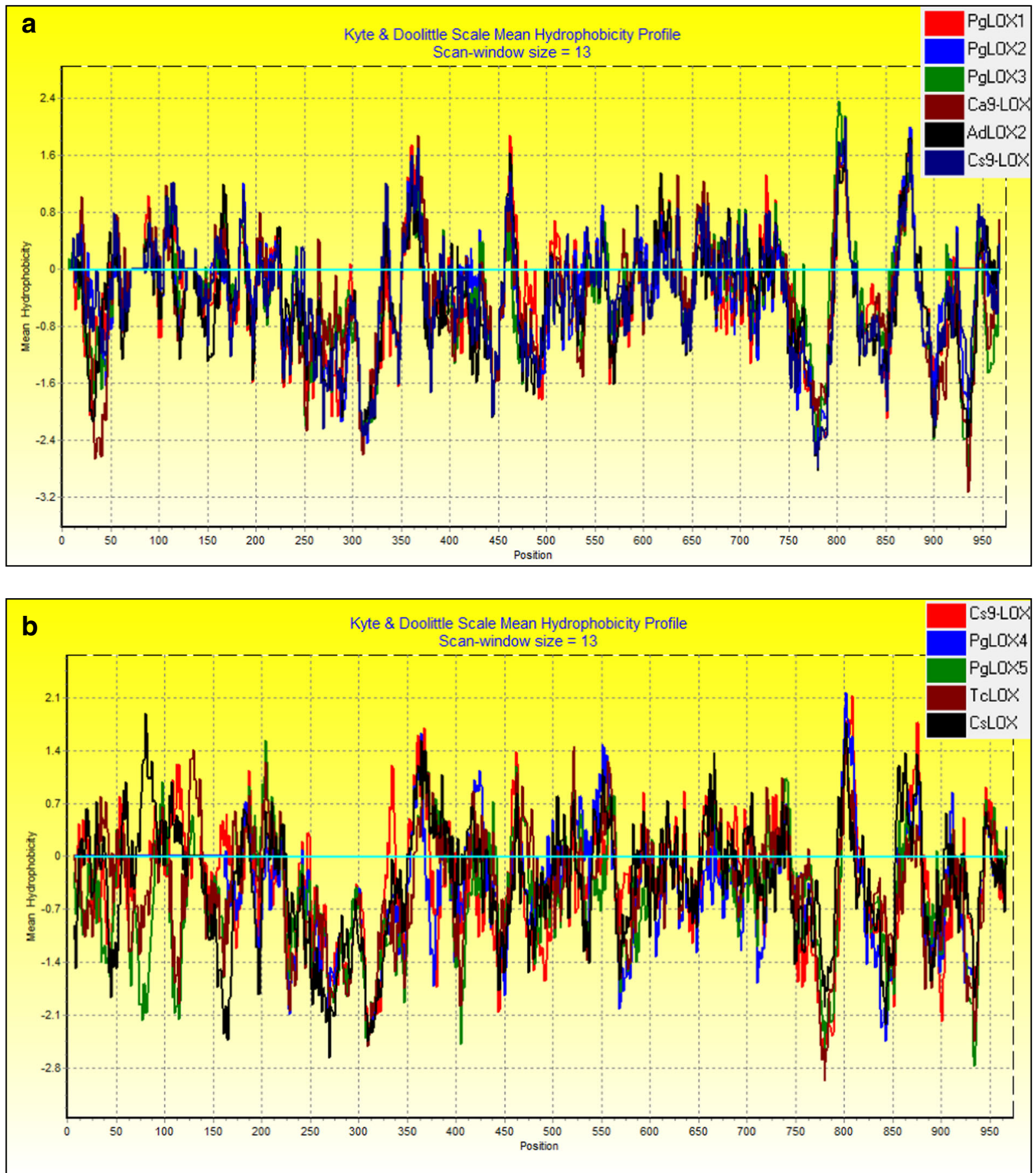

Fig. 3 Superimposed hydrophilicity profiles for 9- and 13-PgLOXs and homologous plant LOXs. Hydrophobic domains are indicated by positive numbers; hydrophilic domains are above the line and hydrophilic domains are below the line

LOX-derived C6 volatiles accumulated in wounded plants and acted as the signal molecule to induce defense and repair mechanisms on the damaged site (Bate and
Rothstein 1998). Also, JA is an essential signaling oxylipin produced highly in wounded plants. There are six LOX genes in tomato (Solanum lycopersicum). 
Table 3 Secondary structure predictions for PgLOXs and homologous plant LOXs

\begin{tabular}{lllll}
\hline Protein & $\alpha$-Helix & $\beta$-Turn & Extended strand & Random coil \\
\hline PgLOX1 & 312 & 74 & 128 & 301 \\
PgLOX2 & 297 & 90 & 146 & 327 \\
PgLOX3 & 264 & 95 & 141 & 338 \\
At9-LOX & 335 & 70 & 149 & 332 \\
PgLOX4 & 268 & 73 & 143 & 278 \\
PgLOX5 & 335 & 77 & 144 & 352 \\
At13-LOX6 & 346 & 62 & 140 & 369
\end{tabular}

TomloxA, TomloxB, and TomloxE encoded 9-LOXs and their functions are not related to C6 biosynthesis (Griffiths et al. 1999; Chen et al. 2004). However, other three chloroplast-localized LOXs of TomloxC, TomloxD and TomloxF involved in generation of JA and C6 volatiles (Chen et al. 2004).

Among all PgLOXs, only PgLOX3, PgLOX4 and $P g L O X 5$ transcriptions were affected by $100 \mu \mathrm{M} \mathrm{MJ}$ in the adventitious roots of $P$. ginseng as suggesting that those LOXs were elicitor-responsive. In most plants, wound-responsive LOXs are induced by JA (Porta et al. 1999) and PgLOX5 which was early responsive to mechanical damage, up-regulated earlier than $P g L O X 4$ by MJ. Endogenous JA biosynthesis can be stimulated by several elicitors and it is thought to be a transduction molecule for production of secondary metabolites (Gundlach et al. 1992). Several studies have reported that exogenously applied jasmonates could induce endogenous JA biosynthesis (Zhao et al. 2005). Our previous study (Rahimi et al. 2014) provided evidence that higher expression of LOX in ginseng roots preceded JA accumulation when elicited. These reports can provide support for our study that the higher induction of $P g L O X$ genes can affect endogenous JA level and it can consequently change the secondary metabolite content by regulating expression level of secondary metabolite biosynthetic genes (Reviewed by Rahimi et al. 2015a; Rahimi et al. 2015b; Devi Balusamy et al. 2015) .

C6 volatiles and JA are the essential molecules for plant response to biotic stress, as it was already shown LOX derived (E)-2-hexenal released from infected leaves with bacteria strongly inhibit Pseudomonas syringae growth (Croft et al. 1993) and the aerial treatment of Arabidopsis with that induced expression of defensive genes (Bate and Rothstein 1998). Higher mRNA levels of $P g L O X s$ during bacterial infection can suggest their role in response to biotic stress. LOX pathway can produce hydroperoxy, hydroxy and keto fatty acids upon infection by pathogen especially during the hypersensitive reaction via LOX action. It was suggested that these LOX pathway products can regulate pathogenesis-related and oxidative-stress-related genes as well as of cell death (Weber 2002). LOX-derived $\alpha$ DOX pathway was induced by Pseudomonas syringae infection in Arabidopsis and $\alpha$-DOX reaction products can be suggested to protect plant tissues from oxidative stress and cell death (De León et al. 2002).

Drought is a severe environmental stress which can cause significant reduction of crops yield. The effect of wounding on expression of LOX genes are well studied whereas less information related the response of LOX genes to water deficit is available. Expression analysis of $P g L O X 3$ in ginseng seedlings subjected to withholding water demonstrated that early stimulation of PgLOX3 expression could correspond to an
Fig. 4 Expression of $P g L O X$ genes in specific organs of 3-year old ginseng. The error bars represent the standard error of the means of three independent replicates

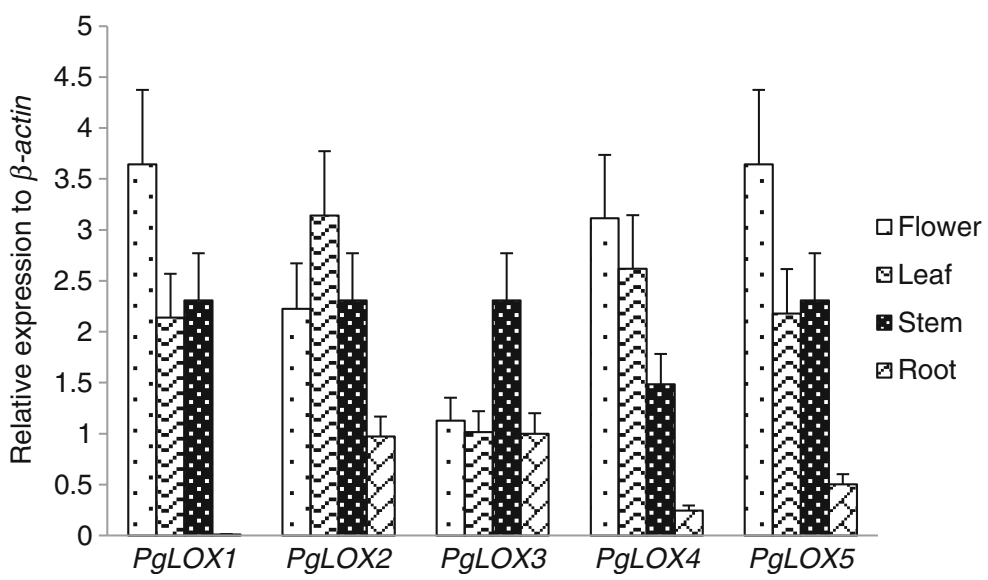



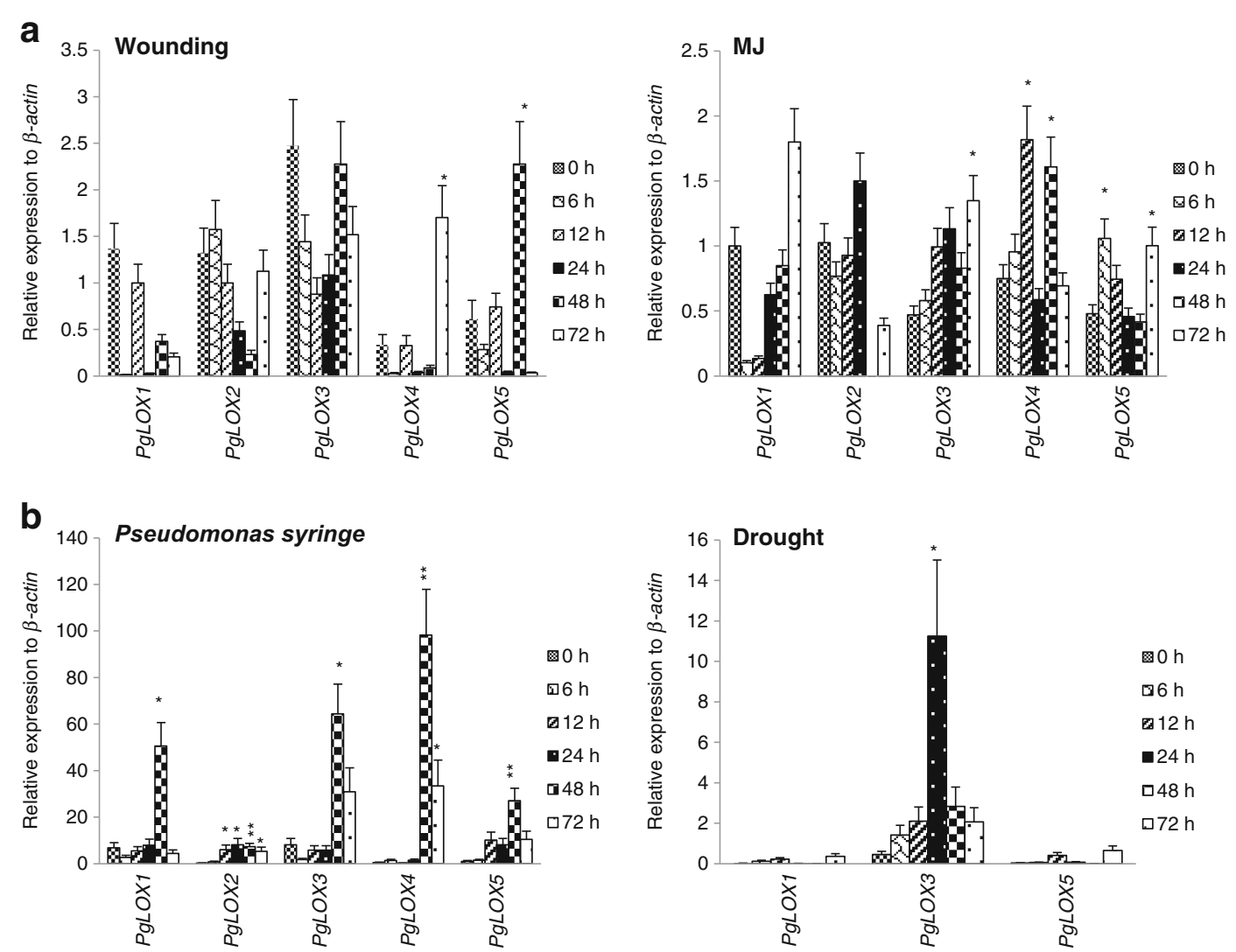

Fig. 5 a Inductive effect of MJ $(100 \mu \mathrm{M})$ and wounding on expression of $P g L O X$ genes in ginseng adventitious roots and 4-week old ginseng seedlings. b Effect of dehydration and infection with Pseudomonas syringe pv tomato strain DC3000 suspension on mRNA level of $P g L O X$. 30-day old ginseng adventitious roots were subject to

adaptive response to drought-stress. This can be correlated with the effect of water deficit on modification of membrane lipids and the immediate induction of LOX activity (Maccarrone et al. 1995). As it was already reported that $\mathrm{C} 6$ volatile virgin olive oil content and composition was affected by water deficit (Gómez-Rico et al. 2006; Servili et al. 2007; Stefanoudaki et al. 2009; Dabbou et al. 2011), it may also suggest that higher mRNA level of $L O X 3$ in drought treated seedlings affect the amount of those related oxylipins. The genomic DNA structure of $P g L O X 3$ revealed a nucleotide sequence with high identity with EST of severe drought-treated Populus encoded the PgPsad2 protein which is localized at downstream of PgLOX3 and this result can suggest that $P g L O X 3$ may involve in drought stress tolerance.

In conclusion, we can suggest a crucial role for $P g L O X S$ in protection of ginseng plant under environmental

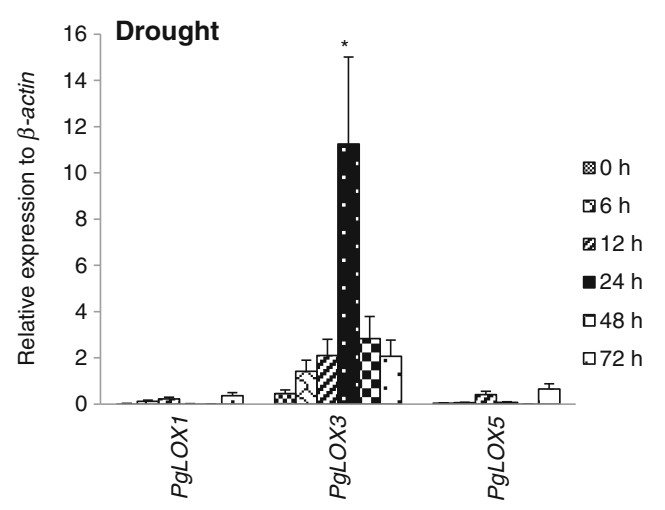

MJ treatments. Four-week old ginseng seedlings were subject to these treatments. $\beta$-actin is an internal reference gene. The error bars represent the standard error of the means of three independent replicates and it was statistically analyzed and compared with control $(* P<0.05 ; * * P<0.01)$ using Student's $t$ test

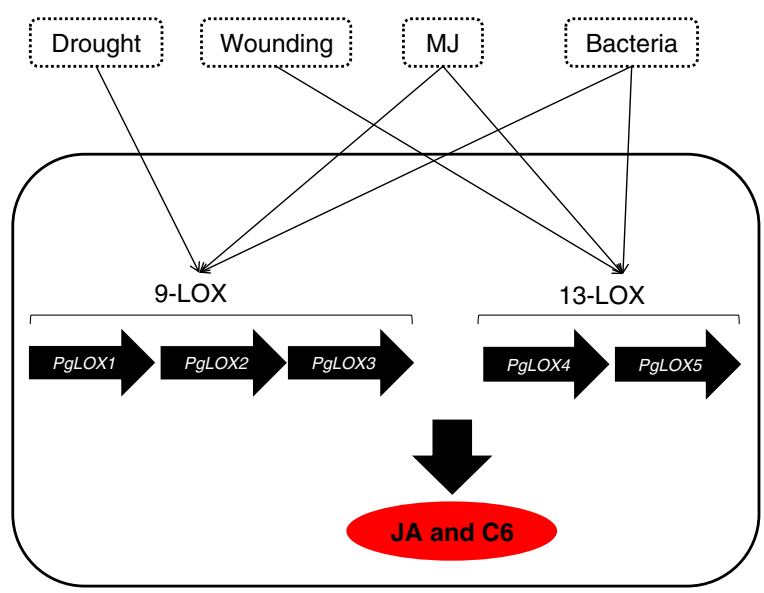

Fig. 6 Presumptive model for activation of $P g L O X$ genes in response to drought, wounding, methyl jasmonate treatment and bacterial infection. Abbrevations: JA, jasmonic acid; LOX, lipoxygenase; MJ, methyl jasmonate 
stresses. However, our results showed that ginseng $L O X$ genes have different expression pattern which may suggest their different specific function against various environmental stresses (Fig. 6), hence further experiments on analysis of $\mathrm{C} 6$ volatiles and JA production in overexpressed and/or knockout lines of PgLOXs can answer the questions related to their function in response to wounding, MJ, bacterial infection and drought stresses. Less information related to the molecular characterization of LOX isoforms in ginseng is available hence; this study might provide support for more analysis of LOXs in ginseng. Further studies on $\mathrm{PgLOX}$ genes are needed for specific localization within the cell and substrate preferences to clarify the physiological functions of each LOX isozymes in ginseng.

Acknowledgments This research was supported by the Basic Science Research Program through the National Research Foundation (NRF) by the Ministry of Education (2013R1A1A2064430), Republic of Korea (YJ Kim) and iPET (312064-03-1-HD040), Korea Institute of Planning and Evaluation for Technology in Food, Agriculture, Forestry and Fisheries, Republic of Korea (DC Yang).

\section{Compliance with ethical standards}

Conflict of interest None.

\section{References}

Andreou, A., \& Feussner, I. (2009). Lipoxygenases-structure and reaction mechanism. Phytochemistry, 70(13), 1504-1510.

Bailey, T. L., Boden, M., Buske, F. A., Frith, M., Grant, C. E., Clementi, L., ... Noble, W. S. (2009). MEME SUITE: tools for motif discovery and searching. Nucleic acids research, gkp335.

Bate, N. J., \& Rothstein, S. J. (1998). C6-volatiles derived from the lipoxygenase pathway induce a subset of defense-related genes. The Plant Journal, 16(5), 561-569.

Blée, E., \& Joyard, J. (1996). Envelope membranes from spinach chloroplasts are a site of metabolism of fatty acid hydroperoxides. Plant Physiology, 110(2), 445-454.

Chen, G., Hackett, R., Walker, D., Taylor, A., Lin, Z., \& Grierson, D. (2004). Identification of a specific isoform of tomato lipoxygenase (TomloxC) involved in the generation of fatty acid-derived flavor compounds. Plant Physiology, 136(1), 2641-2651.

Corbin, J. A., Evans, J. H., Landgraf, K. E., \& Falke, J. J. (2007). Mechanism of specific membrane targeting by $\mathrm{C} 2$ domains: localized pools of target lipids enhance $\mathrm{Ca}^{2+}$ affinity. Biochemistry, 46(14), 4322-4336.

Croft, K. P., Juttner, F., \& Slusarenko, A. J. (1993). Volatile products of the lipoxygenase pathway evolved from
Phaseolus vulgaris (L.) leaves inoculated with Pseudomonas syringae pv phaseolicola. Plant Physiology, 101(1), 13-24.

Dabbou, S., Brahmi, F., Selvaggini, R., Chehab, H., Dabbou, S., Taticchi, A., ... Hammami, M. (2011). Contribution of irrigation and cultivars to volatile profile and sensory attributes of selected virgin olive oils produced in Tunisia. International Journal of Food Science \& Technology, 46(9), 1964-1976.

De León, I. P., Sanz, A., Hamberg, M., \& Castresana, C. (2002). Involvement of the Arabidopsis $\alpha$-DOX1 fatty acid dioxygenase in protection against oxidative stress and cell death. The Plant Journal, 29(1), 61-72.

Devi Balusamy, S. R., Rahimi, S., Sukweenadhi, J., Kim, Y. J., Yang, D. C. (2015). Exogenous methyl jasmonate prevents necrosis caused by mechanical wounding and increased terpenoid biosynthesis in Panax ginseng. Plant Cell, Tissue and Organ Culture, 123(2), 341-348.

Emanuelsson, O., Nielsen, H., \& Von Heijne, G. (1999). ChloroP, a neural network-based method for predicting chloroplast transit peptides and their cleavage sites. Protein Science, $8(05), 978-984$.

Feussner, I., \& Kindl, H. (1992). A lipoxygenase is the main lipid body protein in cucumber and soybean cotyledons during the stage of triglyceride mobilization. FEBS Letters, 298(2), 223-225.

Feussner, I., \& Kindl, H. (1994). Particulate and soluble lipoxygenase isoenzymes. Planta, 194(1), 22-28.

Feussner, I., \& Wasternack, C. (2002). The lipoxygenase pathway. Annual Review of Plant Biology, 53(1), 275-297.

Feussner, I., Hause, B., Vörös, K., Parthier, B., \& Wasternack, C. (1995). Jasmonate-induced lipoxygenase forms are localized in chloroplasts of barley leaves (Hordeum vulgare $\mathrm{cv}$. Salome). The Plant Journal, 7(6), 949-957.

Gasteiger, E., Hoogland, C., Gattiker, A., Wilkins, M. R., Appel, R. D., Bairoch, A. (2005). Protein identification and analysis tools on the ExPASy server. In The proteomics protocols handbook (pp. 571-607). Humana Press.

Geourjon, C., \& Deleage, G. (1995). SOPMA: significant improvements in protein secondary structure prediction by consensus prediction from multiple alignments. Computer applications in the biosciences: CABIOS, 11(6), 681-684.

Gómez-Rico, A., Salvador, M. D., La Greca, M., \& Fregapane, G. (2006). Phenolic and volatile compounds of extra virgin olive oil (Olea europaea L. Cv. Cornicabra) with regard to fruit ripening and irrigation management. Journal of Agricultural and Food Chemistry, 54(19), 7130-7136.

Grayburn, W. S., Schneider, G. R., Hamilton-Kemp, T. R., Bookjans, G., Ali, K., \& Hildebrand, D. F. (1991). Soybean leaves contain multiple lipoxygenases. Plant Physiology, 95(4), 1214-1218.

Griffiths, A., Prestage, S., Linforth, R., Zhang, J., Taylor, A., \& Grierson, D. (1999). Fruit-specific lipoxygenase suppression in antisense-transgenic tomatoes. Postharvest Biology and Technology, 17(3), 163-173.

Gundlach, H., Müller, M. J., Kutchan, T. M., \& Zenk, M. H. (1992). Jasmonic acid is a signal transducer in elicitorinduced plant cell cultures. Proceedings of the National Academy of Sciences, 89(6), 2389-2393.

Heitz, T., Bergey, D. R., \& Ryan, C. A. (1997). A gene encoding a chloroplast-targeted lipoxygenase in tomato leaves is 
transiently induced by wounding, systemin, and methyl jasmonate. Plant Physiology, 114(3), 1085-1093.

Hornung, E., Walther, M., Kühn, H., \& Feussner, I. (1999). Conversion of cucumber linoleate 13-lipoxygenase to a 9lipoxygenating species by site-directed mutagenesis. Proceedings of the National Academy of Sciences, 96(7), 4192-4197.

Howe, G. A., \& Jander, G. (2008). Plant immunity to insect herbivores. Annual Review of Plant Biology, 59, 41-66.

Hwang, I. S., \& Hwang, B. K. (2010). The pepper 9-lipoxygenase gene CaLOX1 functions in defense and cell death responses to microbial pathogens. Plant Physiology, 152(2), 948-967.

Keegstra, K., \& Cline, K. (1999). Protein import and routing systems of chloroplasts. The Plant Cell Online, 11(4), 557570 .

Kyte, J., \& Doolittle, R. F. (1982). A simple method for displaying the hydropathic character of a protein. Journal of Molecular Biology, 157(1), 105-132.

Livak, K. J., \& Schmittgen, T. D. (2001). Analysis of relative gene expression data using real-time quantitative PCR and the 2$\Delta \Delta$ CT method. Methods, 25(4), 402-408.

Maccarrone, M., Veldink, G. A., Agro, A. F., \& Vliegenthart, J. F. (1995). Modulation of soybean lipoxygenase expression and membrane oxidation by water deficit. FEBS Letters, 371(3), 223-226.

Maucher, H., Hause, B., Feussner, I., Ziegler, J., \& Wasternack, C. (2000). Allene oxide synthases of barley (Hordeum vulgare cv. Salome): tissue specific regulation in seedling development. The Plant Journal, 21(2), 199-213.

Murashige, T., \& Skoog, F. (1962). A revised medium for rapid growth and bio assays with tobacco tissue cultures. Physiologia Plantarum, 15(3), 473-497.

Nellen, A., Rojahn, B., \& Kindl, H. (1995). Lipoxygenase forms located at the plant plasma membrane. Zeitschrift für Naturforschung, 50c, 29-36.

Park, S. W., Li, W., Viehhauser, A., He, B., Kim, S., Nilsson, A. K., ... Lawrence, C. B. (2013). Cyclophilin 20-3 relays a 12oxo-phytodienoic acid signal during stress responsive regulation of cellular redox homeostasis. Proceedings of the National Academy of Sciences, 110(23), 9559-9564.

Porta, H., Rueda-Benítez, P., Campos, F., Colmenero-Flores, J. M., Colorado, J. M., Carmona, M. J., ... Rocha-Sosa, M. (1999). Analysis of lipoxygenase mRNA accumulation in the common bean (Phaseolus vulgaris L.) during development and under stress conditions. Plant and cell physiology, 40(8), 850-858.

Rahimi, S., Devi, B. S. R., Khorolragchaa, A., Kim, Y. J., Kim, J. H., Jung, S. K., \& Yang, D. C. (2014). Effect of salicylic acid and yeast extract on the accumulation of jasmonic acid and sesquiterpenoids in Panax ginseng adventitious roots. Russian Journal of Plant Physiology, 61(6), 811-817.

Rahimi, S., Kim, Y. J., Yang, D. C. (2015a). Production of ginseng saponins: elicitation strategy and signal transductions. Applied microbiology and biotechnology, 99(17), 6987-96.
Rahimi, S., Kim, Y. J., Devi, B. S. R., Oh, J. Y., Kim, S. Y., Kwon, W. S., \& Yang, D. C. (2015b). Sodium nitroprusside enhances the elicitation power of methyl jasmonate for ginsenoside production in Panax ginseng roots. Research on Chemical Intermediates. doi:10.1007/s11164-015-2188x.

Royo, J., Vancanneyt, G., Pérez, A. G., Sanz, C., Störmann, K., Rosahl, S., \& Sánchez-Serrano, J. J. (1996). Characterization of three potato lipoxygenases with distinct enzymatic activities and different organ-specific and wound-regulated expression patterns. Journal of Biological Chemistry, 271(35), 21012-21019.

Sathiyamoorthy, S., In, J. G., Gayathri, S., Kim, Y. J., \& Yang, D. C. (2009). Generation and gene ontology based analysis of expressed sequence tags (EST) from a Panax ginseng C.A. Meyer roots. Molecular Biology Reports, 46(7), 932-939.

Scott, R. J., Spielman, M., \& Dickinson, H. G. (2004). Stamen structure and function. The Plant Cell Online, 16(suppl 1), S46-S60.

Servili, M., Esposto, S., Lodolini, E., Selvaggini, R., Taticchi, A., Urbani, S., ... Gucci, R. (2007). Irrigation effects on quality, phenolic composition, and selected volatiles of virgin olive oils cv. Leccino. Journal of Agricultural and Food Chemistry, 55(16), 6609-6618.

Shen, J., Tieman, D., Jones, J. B., Taylor, M. G., Schmelz, E., Huffaker, A., ... Klee, H. J. (2014). A 13lipoxygenase, TomloxC, is essential for synthesis of C5 flavour volatiles in tomato. Journal of experimental botany, 65(2), 419-428.

Stefanoudaki, E., Williams, M., Chartzoulakis, K., \& Harwood, J. (2009). Effect of irrigation on quality attributes of olive oil. Journal of Agricultural and Food Chemistry, 57(15), 70487055.

Stephenson, L. C., Bunker, T. W., Dubbs, W. E., \& Grimes, H. D. (1998). Specific soybean lipoxygenases localize to discrete subcellular compartments and their mRNAs are differentially regulated by source-sink status. Plant Physiology, 116(3), 923-933.

Wasternack, C. (2007). Jasmonates: an update on biosynthesis, signal transduction and action in plant stress response, growth and development. Annals of Botany, 100(4), 681697.

Weber, H. (2002). Fatty acid-derived signals in plants. Trends in Plant Science, 7(5), 217-224.

Yang, X. Y., Jiang, W. J., \& Yu, H. J. (2012). The expression profiling of the lipoxygenase (LOX) family genes during fruit development, abiotic stress and hormonal treatments in cucumber (Cucumis sativus L.). International journal of molecular sciences, 13(2), 2481-2500.

Zhao, J., Davis, L. C., \& Verpoorte, R. (2005). Elicitor signal transduction leading to production of plant secondary metabolites. Biotechnology Advances, 23(4), 283-333. 